\title{
Revealing the truth about nutrition labelling: The age of the confused
}

\section{consumer}

\section{Kassandra Wagner}

Department of Food Science, Ontario Agricultural College, University of Guelph, Guelph, ON Canada. Faculty supervisor: Dr. Rickey Yada. For correspondence, please email: kassandra.wagner@live.ca.

\begin{abstract}
Inadequate nutrition is considered to be a leading cause of mortality in the world. Access to quality information about the nutritional quality of food products is essential for making informed decisions in terms of food selection. To this end, nutrition labelling can be used as a tool for helping guide consumers food choices. This review article will examine which consumers use nutrition labels and what types of information they get from them; how nutrition labels influence health choices, dietary habits, and consumer behaviours; and finally, current practices in nutrition labelling and whether this aligns with consumer preferences. Findings from this article are useful for developing methods of nutritional label education and understanding how nutrition labels can be improved to become more useful to consumers.
\end{abstract}

Keywords: health; nutrition labelling; food products; packaging; public education; consumer perception

\section{Introduction}

Inadequate nutrition is considered to be a leading cause of mortality in the world. The World Health Organization (2002) states that five of six risk factors for substandard health conditions are connected to diets poor in nutrients. Access to quality information about the nutritional quality of food products is essential for making informed decisions in terms of food selection. To this end, nutrition labelling can be used as a tool for helping guide consumers food choices. This review article will examine which consumers use nutrition labels and what types of information they get from them; how nutrition labels influence health choices, dietary habits, and consumer behaviours; and finally, current practices in nutrition labelling and whether this aligns with consumer preferences. Findings from this article are useful for developing methods of nutritional label education and understanding how nutrition labels can be improved to become more useful to consumers.

\section{Consumer Use of Nutrition Labels}

Even though consumers can interpret basic information on nutrition labels, for example the amount of a nutrient in a given food product, more complex information is often seen as unclear and confusing (Cowburn \& Stockley 2004; Higginson et al. 2002). Cowburn and Stockley (2004) conducted a systematic analysis of 103 published and unpublished studies on consumer understanding in Europe and parts of North America. The research showed that technical and numerical information on nutrition labels was accountable for most of the perplexities. Consumers stated that terms such as fat, vitamins, calories, salt and sugar were easy to understand; however, certain concepts - for example, the relationship between energy and calories, salt and sodium levels, carbohydrates and sugars, and fatty acids and cholesterol - were more difficult to understand.

In Cowburn and Stockley's (2004) study, consumers who were older or had lower levels of education or income were found to have the most challenges with interpreting information. Rothman et al. (2006) completed a crosssectional study on primary care patients that found that weak numeracy and literacy skills were major factors contributing to poor understanding of nutritional labels. A controlled experimental evaluation of multiple labelling formats by Levy and Fein (1998) revealed that men tended to have a lower interest in reviewing nutrition labels than women. Sullivan and Gottschall-Pass (1995) showed that racial groups including African-Americans, Asians, and Hispanics were less likely than Caucasian individuals to read and interpret nutrition labels; this was found to be a result of limited time, low literacy levels and a lack of understanding of nutrition labelling.

Consumers most frequently make mistakes in assessing nutrient amounts from recommended intake levels. Older consumers and those with lower levels of education or income had the most trouble translating information from 
100 grams to gram per serving and serving size (ByrdBredbenner et al. 2001). Percentage energy was also another concept that was not very well recognized. Easy to read verbal descriptors of nutrient content (e.g., "high," "medium," or "low") as well as the inclusion of recommended reference values might help address these issues (European Heart Network 2003). For example, studies have shown that the nutrition value of a product is more easily interpreted when a reference point is given $(\mathrm{Li}$ et al., 2002). The presentation of daily reference standards and brand goals beside the verbal and graphical presentations has previously been found to be helpful. This allows consumers to use numerical information to make simple comparisons between products ( $\mathrm{Li}$ et al. 2002). Consumers tend to make more accurate comparisons between products when the products have similar ingredients that can be compared. When products are dissimilar, consumers find comparisons more challenging and as a result will often use a single nutrient as a criterion for assessing an entire product (Sullivan and Gottschall-Pass 1995). Numerical and nonnumerical aids may help consumers to compare products more accurately. Verbal descriptors displayed beside numerical information have been shown to be the most helpful (Higginson et al. 2002).

Consumers are capable of using numerical information found on nutrition labels to perform simple calculations. Levy, Fein and Schucker (1991) recruited 1,216 subjects over the age of 18 in eight shopping malls across the United States to review nutrition label data and perform relatively simple calculations with it; for example, participants were asked to determine the amount of a nutrient present in a serving of a product. Consumers were able to use numerical information somewhat accurately to complete simple calculations; however, their degree of accuracy decreased as the complexity of the tasks increased. Unfamiliarity with the label format, lower levels of education, and increasing age were also determining factors. Non-numerical evaluation aids like bar charts or pie charts were seen as beneficial to consumers performing the simple calculations. Including a numerical reference, such as the percentage of dietary reference values or a guideline of daily amounts used, was most helpful to consumers. Non-numerical labelling was also advantageous. While consumers preferred bar charts, they were found to be most precise when reading verbal descriptors in more objective evaluations of nutrition labelling.

\section{Consumer Choices}

Nutritional labels on food products were developed to improve and protect the public's health by displaying nutrition information for consumers to make informed dietary choices. The healthiness of a consumer decision is influenced by label use. Drichoutis et al. (2005) showed that there is a positive connection between nutrition label use and purchase behavior. Regardless of the type of nutrition label, the overall quality of consumer diets was increased by use of the label.

A study by Lin, Lee and Yen (2004) showed that individuals who consume more total fat, saturated fat or cholesterol are less likely to search for information on nutrition labels in comparison to those who consumer healthier diets. These results revealed that some individuals choose to ignore information that contradicts their preferred eating habits. Reviewing labels are also related to how highly one values nutrition, their understanding of nutrition, and their awareness of overconsumption and associated health issues (Kreuter et al. 1997). Behavioural traits and psychosocial influences such as moderate physical activity, confidence in the relationship between diet and cancer, a desire to eat healthy, and trying to prevent weight gain were also positively related to nutrition label use (Kreuter et al. 1997).

Lin, Lee and Yen (2004) examined results from the Diet and Health Knowledge Survey and the Continuing Survey of Food Intakes completed by Americans over the age of 20 in 1994 to 1996. Their research concluded that consumer education on how to interpret nutrition labels was helpful in helping consumers make knowledgeable decisions. However, reassuring consumers to take full advantage of labelling can be difficult for nutrition educators. New and unique education methods will be required to address consumers; thus, it will be essential to determine what an optimal nutrition label would look like in order to help consumers use it to its full potential.

\section{Food Label Standardization}

There are many ways that food can be labelled, some easier to understand and interpret than others; thus it is important to investigate the labelling preferences of consumers. An alternative method of labelling is known as the front-of-package (FOP) system. This method has the ability to be standardized and used on all food and beverage products. The very first FOP symbols to appear on grocery products were created by non-profit organizations. Examples include the "Heart Check" symbol, which was developed by the American Heart Association (AHA) to show consumers when a food was "heart friendly." The "Keyhole" symbol was developed in Sweden to help consumers make more informed health choices and is used on products that contain low amounts of total fat, saturated fat, salt, and sugar (National Food Agency 1989). The Canadian Heart and Stroke Foundation (1999) created the "Health Guide" program, which is similar to the AHA's and Sweden's initiatives. All three of these FOP variations display a single symbol on the food products that qualify. However, the downfalls to all three of these FOP symbols is that there is not an accepted industry-wide rating method and approach; therefore, these synbols may not be as helpful for encouraging healthy consumer choices as other systems of labelling (Wartella et al. 2010). Also, they do not take other 
important nutrients into account such as vitamins or protein. Creating a single, dependable FOP nutrition labelling system will diffuse some of this confusion, particularly because the use of multiple labels that are different from each other may be confusing to customers.

The United Kingdom has accepted a front-of-package labelling method known as the "Traffic Light System" (TLS) that displays the amounts of fats, sugars, saturates, and salt in a particular food item (Food Standards Agency 2007). A red light means that the food contains a very high level of a given nutrient whereas amber indicates medium amounts and green is used for low levels. The color is dependent on the quantity of each of the nutrients per 100 gram of a food, which can then be converted into a per portion amount. The "Traffic Light System" was implemented in response to consumers' poor understanding of previous nutritional labelling and aims to help them make informed dietary choices. Unlike previous labelling systems, consumers in the United Kingdom find TLS extremely simple to understand. However, public health and consumer acceptance for implementing this method has been a challenge with the food industry, where uptake and acceptance has been much slower. The success of TLS lies in its simplicity, as more complicated nutrition labels can make product choices difficult for consumers (Food Standards Agency 2007).

Feunekes et al. (2008) studied consumer acceptance of FOP nutrition labelling using the TLS system as a test case. The researchers recruited 1,630 women and men between the ages 18 to 55 in the United Kingdom, Italy, Netherlands, and Germany to complete an online questionnaire that examined consumers' perceptions of three food products based on their food labels. TLS was found to perform generally well in informing consumers' sense of liking, apprehension, and validity, but not perceived healthiness. This may be the result of subjects' interpretations of the red, amber, and green criteria, which may differ from what was intended by the labellers. Additionally, the TLS method has been criticized for not including enough information on its labels about beneficial nutrients such as fibre or protein (European Heart Network 2003). Moreover, the labels can be confusing in some contexts, for example when a product receives two green lights and two red lights. This makes it difficult to rate the product's overall healthiness. Also, some foods that are considered healthy in reasonable quantities, such as cheese, may have a red light for certain ingredients (e.g., salt) that could discourage consumers from buying the product altogether (European Heart Network 2003). Although the TLS labelling system has some limitations, it remains a way of labelling food that is easily understandable to consumers. Due to its success in Europe, it has already achieved a strong influence on food companies. For instance in the United Kingdom, cereals possessing green lights or amber lights are increasing twice as fast as breakfast cereals that have red lights (Chun Yu Louie et al. 2008).

The most recent development in FOP labelling was accomplished by America's leading food and beverage manufacturers and retailers (National Research Council
2012). The "Nutrition Keys" method provides a summary of essential information from the label like calories, saturated fat, sodium, and total sugars content on the front surfaces of the packages. For some food and beverage products, the "Nutrition Keys" icon provides information about "nutrients to encourage," such as potassium, fiber, vitamin A, vitamin C, vitamin D, calcium, iron, and protein. Although all of the FOP systems have different methods of grading food or beverages, the main goal is to give the consumer a simple-tounderstand label that indicates whether the product is a healthy choice in comparison to others within a category (Dötsch-Klerk and Jansen 2008).

Overall, FOP systems can be categorized into three main groups: (1) nutrient-specific systems such as percent daily value or TLS; (2) single-symbol summary indicator systems such as AHA's "Heart Check" or Sweden's "Keyhole" symbol; and (3) food group information systems that involve symbols depending on the amount of a food group or food ingredient such as the "Whole Grain Stamp" (Institute of Medicine 2010). Creating an environment that supports consumers in making informed decisions is fundamental for advocating good health. Nutrition education can be a tool for aiding the consumer to understand information on food labels. For example, motivational messages (e.g., in the form of advertisements or in the media) focusing on healthy eating, managing a healthy weight, consuming foods low in sodium, saturated fats, etc. can be useful in educating consumers about good nutrition. Another benefit of messaging is that it can help teach consumers the technical skills needed to utilize a food label to its full potential (Kreuter et al. 1997). Dietitians also have a leading role in educating the public with knowledge of nutrition and labelling information (American Heart Association 2009). These professionals should enforce the concept of a proper energy balance through personal dietary counseling, clinical practice or community-based interventions (e.g., assessment of nutritional messages from food and beverage marketing in schools).

\section{Conclusion}

Nutrition labels can be useful for helping consumers make healthy and informed food selections. Labelling is convenient as it administers information about the nutrient content of a food; however, it is only useful when the consumer can interpret the messages or information provided. Individuals who use labels are more likely to have a higher level of educational achievement, interest, higher income and of the female gender. There is a positive correlation between nutrition label use and purchase behavior; this was dependent on the impact of nutritional label on the consumers' values and perception of health. A basic background of nutrition or interest in maintaining a healthy diet will contribute to knowledgeable food purchases. Nutrition education can be a wonderful asset for encouraging nutrition label understanding and accuracy during reviewing. There are a variety of tools in which this can be 
accomplished; examples include Front of Package (FOP). The most efficient and easy to use method by consumers was found to be a FOP system that highlighted certain food groups. Nutrition professionals are also an essential part of educating the public.

\section{Acknowledgements}

I would like to express my gratitude to everyone who helped me with this project. I have thoroughly enjoyed having the opportunity to study this topic in detail. A special thanks to Dr. Rickey Yada for all his support and guidance as my faculty supervisor. Also, I would like to recognize Dr. Alison Duncan from the Human Health and Nutritional Sciences department for her assistance.

\section{References}

American Heart Association. 2009. Implementing American Heart Association pediatric and adult nutrition guidelines. Circulation. 119(8): 1161-1175.

Byrd-Bredbennr C, Alfieri L, Wong A, Cottee P. 2001. The inherent educational qualities of nutrition labels. Family and Consumer Sciences Research Journal. 29(3): 265-280.

Canadian Heart and Stroke Foundation. 1999. Health check. Retrieved on November 9, 2014 from http://www.healthcheck.org/page/canadas-foodguide

Chun Yu Louie J, Flood V, Rangan A, Hector D, Gill T. 2008. A comparison of two nutrition signposting systems for use in Australia. Public Health Nutrition. 19: 121-126.

Cowburn G, Stockley, L. 2004. Consumer understanding and use of nutrition labeling: A systematic review. Public Health Nutrition. 8(1): 21-28.

Dötsch-Klerk M, Jansen L. 2008. The choices programme: A simple, front-of-pack stamp making healthy choices easy. Asian Pacific Journal of Clinical Nutrition, 17: 383-386.

European Heart Network (EHN). 2003. Response on behalf of the European Heart Network to a request for information in view of the revision of Council Directive 90/496/EEC on Nutrition Labeling. Retrieved on November 23, 2013 from http://ec.europa.eu/food/food/labellingnutrition/n utritionlabel/ehn_en.pdf

Feunekes G, Gortemaker I, Willems A, Lion R, van den Kommer, M. 2008. Front-of-pack nutrition labeling: testing effectiveness of different nutrition labeling formats front-of-pack in four European countries. Appetite. 50(1): 57-70.

Food Standards Agency. 2007. Front of pack traffic light signpost labeling - technical guide.

Retrieved on November 11, 2013 from http://www.food.gov.uk/multimedia/pdfs/frontofp ackguidance2.pdf

Higginson C, Rayner M., Draper, S, Kirk TR. 2002. The nutrition label - which information is looked at? Nutrition and Food Science. 32(3): 92-99.

Institute of Medicine. (2010). Examination of front-ofpackage nutrition rating systems and symbols: Phase I report. Washington DC: Institute of Medicine.

Kreuter MW, Brennan LK, Scharff D, Lukwago SN. 1997. Do nutrition label readers eat healthier diets? Behavioral correlates of adults' use of food labels. American Journal of Preventative Medicine. 13: 277-283.

Levy A, Fein SB, Schucker RE. 1991. Nutrition labeling formats: performance and preference. Food Technology. 8: 116-121.

Levy A, Fein S. 1998. Consumers' ability to perform tasks using nutrition labels. Journal of Nutrition Education. 30(4): 210-217.

Li F, Miniard PW, Barone MJ. 2002. The facilitating influence of consumer knowledge on the effectiveness of daily value reference information. Journal of the Academy of Marketing Science, 28(3): 425-436.

Lin C, Lee J, Yen S. 2004. Do dietary intakes affect search for nutrient information on food labels? Social Science and Medicine. 59(9): 1955-1967.

National Food Agency. 1989. Keyhole symbol packaging. Retrieved on November 6, 2014 from http://www.slv.se/en-gb/Group1/Food-andNutrition/Keyhole-symbol/Rules-for-the-keyholesymbol/

National Research Council. 2012. Front-of-package nutrition rating systems and symbols: Promoting healthier choices. Washington, DC: The National Academies Press.

Rothman R, Housam R, Weiss H, Davis D, Gregory R, Gebretsadik T, Shintani A, Elasy T. 2006. Patient understanding of food labels: role of literacy and numeracy. American Preventative Medicine. 31(5), 391-398. 
Sullivan AD, Gottschall-Pass KT. 1995. Food label nutrition literacy: tool development and assessment. Journal of the Canadian Dietetic Association. 56(2), 68-72.

Wartella E, Lichtenstein A, Boon, C. (2010). Phase I report: Examination of front-of-package nutrition rating systems and symbols. Washington, DC: National Academies Press.
World Health Organization (WHO) (2002). The world health report 2002 - reducing risks, promoting healthy life. Geneva, CH: World Health Organization. 